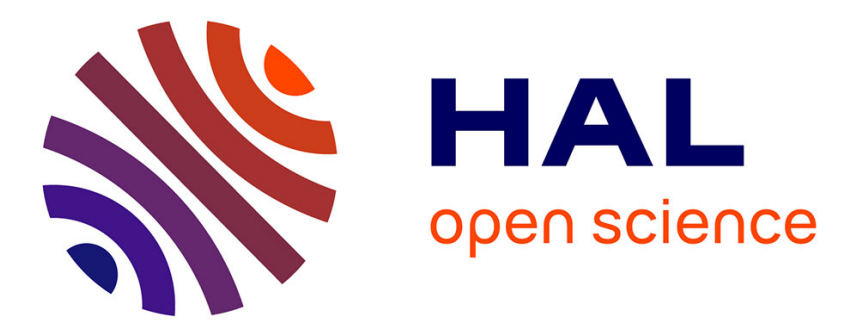

\title{
A third-order sliding-mode observer for a continuous delay chaotic system
}

Octaviana Datcu, Leonid Fridman, Jean-Pierre Barbot

\section{To cite this version:}

Octaviana Datcu, Leonid Fridman, Jean-Pierre Barbot. A third-order sliding-mode observer for a continuous delay chaotic system. IFAC Conference on analysis and control of chaotic systems CHAOS 2012, Jun 2012, Cancun, Mexico. hal-00750616

\section{HAL Id: hal-00750616 https://hal.inria.fr/hal-00750616}

Submitted on 11 Nov 2012

HAL is a multi-disciplinary open access archive for the deposit and dissemination of scientific research documents, whether they are published or not. The documents may come from teaching and research institutions in France or abroad, or from public or private research centers.
L'archive ouverte pluridisciplinaire HAL, est destinée au dépôt et à la diffusion de documents scientifiques de niveau recherche, publiés ou non, émanant des établissements d'enseignement et de recherche français ou étrangers, des laboratoires publics ou privés. 


\title{
A third-order sliding-mode observer for a continuous delay chaotic system
}

\author{
O. DATCU ${ }^{*}$, L. FRIDMAN ${ }^{* *}$, J. P. BARBOT ${ }^{* * *}$ \\ * Politehnica University of Bucharest, Faculty of Electronics, Telecommunications, and \\ Information Technology and Electronic and Control Systems Laboratory (ECS-Lab EA 3649), \\ ENSEA, 6 Avenue du Ponceau, 95014 Cergy-Pontoise Cedex. \\ ** Departamento de Ingenieria de Control y Robotica, Division de Ingenieria Electrica, \\ Facultad de Ingenieria UNAM. \\ *** Electronic and Control Systems Laboratory (ECS-Lab EA 3649), ENSEA, 6 Avenue du \\ Ponceau, 95014 Cergy-Pontoise Cedex and EPI Non-A, INRIA
}

\begin{abstract}
This paper aims firstly to highlight the possibility of recovering a message included in a chaotic continuous time delay system, secondly to show that it is possible to use the third order sliding mode in order to recover directly all the states and the unknown input (message), thirdly to illustrate the robustness of the proposed observer with respect to a noisy signal. This work is based on the concept of left invertibility and recent advances in sliding mode observers. Some theoretical recalls and assumptions are done previously to the presentation of the observer design and the simulation results.
\end{abstract}

\section{INTRODUCTION}

This paper does not aim to deal with the secure data transmission by synchronization of chaotic delayed systems, even if the thread of the article is a transmitter, a receiver and a public channel. Here, the main purpose is to show that it is possible to solve directly a left invertibility problem for chaotic delayed systems with homogenous sliding mode differentiator [Levant(2003)]. In order to consider a very simple example, a two dimension delayed system is presented and a homogeneous sliding mode observer of just third order [Levant(2003)] is used. Moreover, the observer structure is slightly modified in order to resolve the left invertibility problem and some low pass filters are added in order to take into account the noisy output measurement, due to the noise in the public channel. The academical secure data transmission based on chaos synchronization used in order to highlight the efficiency of high order sliding mode observers is represented in Fig. 1 and corresponds to the so called inclusion method [Djemai(2005)].

The robustness of this academical example with respect to known plain text attack is not the goal of the paper, nevertheless it is important to mention that it is generally more difficult to identify delay dynamical systems than classical dynamical systems. The paper is organized as follows: in the next section the proposed chaotic delayed system is presented. In section three some recalls on high order sliding mode are given and the proposed observer is introduced. The last section is dedicated to the presentation and the analysis of the simulation results. The conclusions and the perspectives conclude the paper.

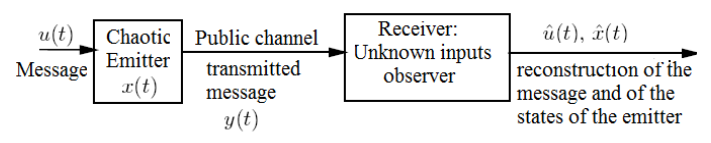

Fig. 1. Chaos synchronisation by means of high order sliding mode observers, using the inclusion of the secret messsage in the dynamics of the chaotic emitter. 


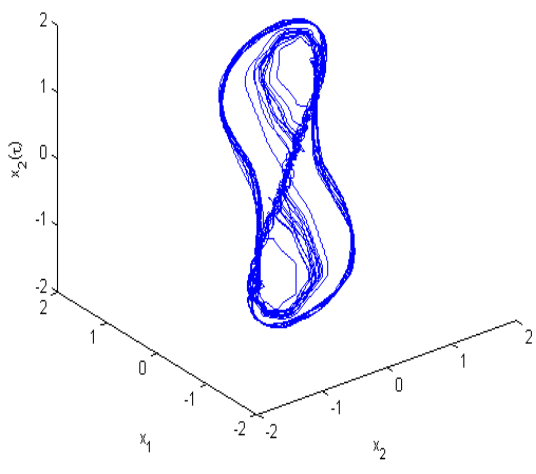

Fig. 2. The states $x_{1}, x_{1}(\tau), x_{2}$ for $u=0$. The initial conditions are: $x_{1}(0)=0, x_{2}(0)=$ 0.1 and $x_{2}(t)=0, \forall t \in[-\tau, 0)$.

\section{A CHAOTIC DELAYED TRANSMITTER}

One of the main advantages of delay systems is the possibility to generate a chaotic behavior with a state dimension smaller than three. The following chaotic delay system, strongly inspired by [Uçar(2003)], is proposed as transmitter:

$$
\begin{aligned}
\dot{x}_{1} & =x_{2}-x_{1} \\
\dot{x}_{2} & =x_{2}(\tau)-x_{2}^{3}(\tau)+0.5\left[1+\left(\frac{x_{2}}{10}\right)^{2}\right] u \\
y & =x_{1}
\end{aligned}
$$

where $x=\left(x_{1}, x_{2}\right)^{T}$ is the state, $y$ is the output, and $u$ is the message. Moreover, $x_{i}$ denotes the state $x_{i}$ at time $t, x_{i}(\tau)$ denotes the state $x_{i}$ at time $t-\tau$, the initial conditions are $x_{1}(0)$ and the function $x_{2}(t)$, defined on $[-\tau, 0]$. The behavior of system (1) for $u$ equal to zero is chaotic as shown in Fig. 2, where the three dimensional state space behavior $\left(x_{1}, x_{1}(\tau), x_{2}\right)$ is given for $\tau=1.6 s$. We have given the state portret in three dimension in order to highlight the key-role of the delay. Setting a message of the form:

$$
u(t)=A \sin (\omega t)
$$

with $A=0.5 \mathrm{~V}$ and $\omega=2 \pi f$, where $f=$ $1 \mathrm{~Hz}$, the chaotic state space behavior of $\left(x_{1}, x_{1}(\tau), x_{2}\right)$ is preserved as shown in Fig. 3 .
Remark 1. The preservation of the chaotic behavior is the first restriction on the message; another restriction is that the message must not be detectable from the output by a simple frequency analysis. Other restrictions will be given in the next section. Obviously, it is also possible to send binary messages, if the amplitude of such messages is small enough to preserve the chaotic behavior.

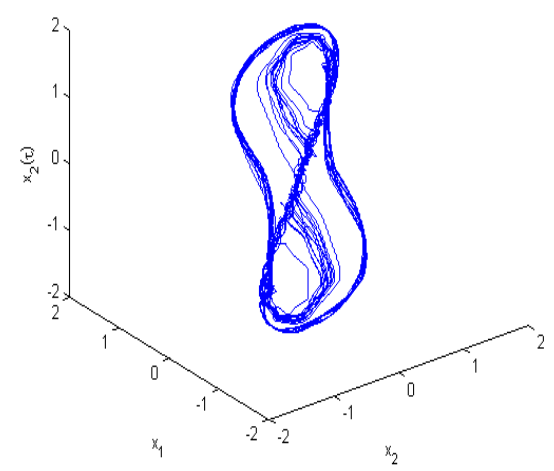

Fig. 3. The states $x_{1}, x_{1}(\tau), x_{2}$ for $u=$ $0.5\left[1+\left(\frac{x_{2}}{10}\right)^{2}\right]$. The initial conditions are: $x_{1}(0)=0, x_{2}(0)=0.1$ and $x_{2}(t)=0, \forall t \in$ $[-\tau, 0)$.

The state $x_{1}$ of the transmitter (1) is presented with the message included in the evolution of the delayed chaotic system in Fig. 4.

\section{A THIRD ORDER SLIDING MODE OBSERVER AS THE RECEIVER OF THE SECRET MESSAGE}

At the reception, the ciphertext, $y$, is represented by the first state of the transmitter (1). A high-order sliding mode observer, (4), is used in order to recover the states $x_{1}$ and $x_{2}$ of the transmitter and the secret message $u$ included in its evolution, when knowing only its output $x_{1}$. The presented proposed observer is based on the so-called real-time exact robust HOSM differentiator [Levant(2006)]. Considering the output $y(t)$ at least $\mathcal{C}^{k}$ (at least $k$ times differentiable) the $\mathrm{k}$-differentiator (i.e. recovering $\dot{y}, \ddot{y}, \ldots, y^{(k)}$, from the measurement of $y$ ) has the following form: 


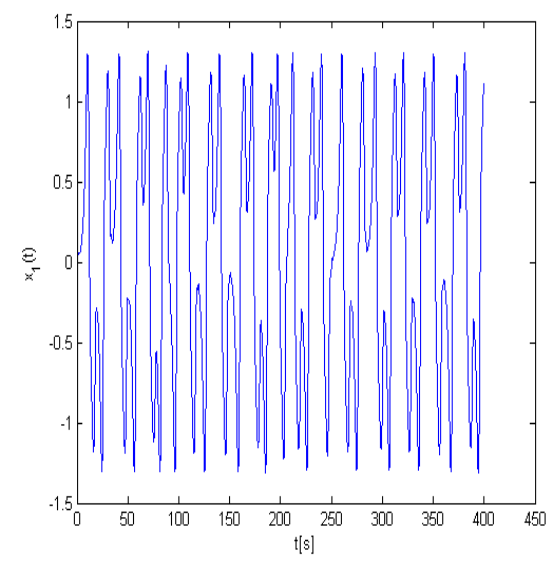

Fig. 4. The output of the transmitter with the message included in the evolution of the delayed chaotic transmitter. The initial conditions are: $x_{1}(0)=0, x_{2}(0)=0.1$ and $x_{2}(t)=0, \forall t \in[-\tau, 0)$.

$$
\begin{aligned}
& \dot{z}_{0}=\nu_{0} \\
& \nu_{0}=z_{1}-\lambda_{k} M^{\frac{1}{k+1}}\left|z_{0}-y\right|^{\frac{k}{k+1}} \operatorname{sign}\left(z_{0}-y\right) \\
& \dot{z}_{1}=\nu_{1} \\
& \nu_{1}=z_{2}-\lambda_{k-1} M^{\frac{1}{k}}\left|z_{1}-\nu_{0}\right|^{\frac{k-1}{k}} \operatorname{sign}\left(z_{1}-\nu_{0}\right) \\
& \vdots \\
& \dot{z}_{k-1}=\nu_{k-1} \\
& \nu_{k-1}=z_{k}-\lambda_{1} M^{\frac{1}{2}}\left|z_{k-1}-\nu_{k-2}\right|^{\frac{1}{2}} . \\
& \quad \quad \operatorname{sign}\left(z_{k-1}-\nu_{k-2}\right) \\
& \dot{z}_{k}=-\lambda_{0} M \operatorname{sign}\left(z_{k}-\nu_{k-1}\right)
\end{aligned}
$$

In the present case a third order HOSM differentiator is designed. This implies that $y$ is at least $\mathcal{C}^{3}$. Moreover, as the observer must reconstruct the states and the secret message $u$, using only the knowledge of $y$, an extended state $x_{3}=0.5\left[1+\left(\frac{x_{2}}{10}\right)^{2}\right] u$ is needed in order to recover also the unknown input. Obviously, this dynamical extension implied some assumptions on the unknown input. Hereafter, all hypotheses on $u$ are summarized:

Hypothesis 1.

- $u$ must be sufficiently small in order to preserve the chaotic behavior of (1).

- $u$ must be sufficiently small and within the frequency bandwidth of the transmitter in order not to be detectable by output frequency analysis.
- $u$ must be at least $\mathcal{C}^{1}$ and both $u$ and its derivative must be bounded.

Finally, the proposed observer has a slightly different form from (3) in order to reduce the gain $M$, and taking into account the structure of (1):

$$
\begin{aligned}
\dot{\hat{x}}_{1}= & \hat{x}_{2}-y-3 M^{1 / 3}\left|\hat{x}_{1}-y\right|^{2 / 3} \\
& \operatorname{sign}\left(\hat{x}_{1}-y\right) \\
\dot{\hat{x}}_{2}= & \hat{x}_{2}(\tau)-\hat{x}_{2}^{3}(\tau)+\hat{x}_{3}-1.5 M^{1 / 2} \\
& \left|\hat{x}_{2}-\nu_{0}\right|^{1 / 2} \operatorname{sign}\left(\hat{x}_{2}-\nu_{0}\right) \\
\dot{\hat{x}}_{3}= & \frac{\hat{x}_{2}}{50}\left(\hat{x}_{2}(\tau)-\hat{x}_{2}^{3}(\tau)+\hat{x}_{3}\right) \frac{\hat{x}_{3}}{1+\left(\frac{\hat{x}_{2}}{10}\right)^{2}} \\
& -1.1 M \operatorname{sign}\left(\hat{x}_{3}-\nu_{1}\right)
\end{aligned}
$$

where

$$
\begin{aligned}
& \nu_{0}=\hat{x}_{2}-3 M^{1 / 3}\left|\hat{x}_{1}-y\right|^{2 / 3} \operatorname{sign}\left(\hat{x}_{1}-y\right) \\
& \nu_{1}=\hat{x}_{3}-1.5 M^{1 / 2}\left|\hat{x}_{2}-\nu_{0}\right|^{1 / 2} \operatorname{sign}\left(\hat{x}_{2}-\nu_{0}\right)
\end{aligned}
$$

Proposition 1. If the initial conditions of system (1) are in the attraction basin of the strange attractor, and if all conditions in the hypothesis 1 are verified, then there exists $M_{0}>0$ such that $\forall M \geq M_{0}$, observer (4) resolves the left invertible problems for system (1) (i.e. the recovering of $x_{2}$ and $u$ ).

Sketch of convergence proof:

Denoting $e:=x-\hat{x}$, one sliding manifold has

$$
\begin{aligned}
& 0=e_{2}-3 M^{1 / 3}\left|e_{1}\right|^{2 / 3} \operatorname{sign}\left(e_{1}\right) \\
& 0=e_{3}-1.5 M^{1 / 2}\left|e_{2}\right|^{1 / 2} \\
& 0=0.5\left[1+\left(\frac{x_{2}}{10}\right)^{2}\right] \dot{u}-1.1 M \operatorname{sign}\left(\hat{x}_{3}-\nu_{1}\right)
\end{aligned}
$$

For convergence analysis with the homogeneity argument see [Levant(2003)], [Levant(2005)] and for noise robustness see [Ángulo(2011)]. From (5), one obtains

$$
\begin{aligned}
& x_{2}=\hat{x}_{2}-3 M^{1 / 3}\left|e_{1}\right|^{2 / 3} \operatorname{sign}\left(e_{1}\right)=\nu_{0} \\
& x_{3}=\hat{x}_{3}-1.5 M^{1 / 2}\left|e_{2}\right|^{1 / 2}=\nu_{1}
\end{aligned}
$$

Consequently, from the definition of $x_{3}$, it can be concluded that:

$$
u=\frac{\hat{x}_{3}}{0.5\left[1+\left(\frac{\hat{x}_{2}}{10}\right)^{2}\right]}
$$


This ends the sketch of proof.

\section{EXPERIMENTAL RESULTS.}

In order to prove the performance of the proposed observer, simulations were done in Simulink and relevant results were obtained. The simulation was done over $400 \mathrm{~s}$, with a fixed sampling step of $T_{s}=5 \cdot 10^{-6} \mathrm{~s}$, using the first order Euler's method in order to solve the ordinary differential equations (ODE) describing the dynamics of the transmitter (chaotic delayed continuous system) and of the receiver (high-order sliding mode observer). On the public channel, the output $y=x_{1}$ of the transmitter reaches the receiver (4). The estimations of the states and of the unknown input are presented in two cases:

- noise-free channel.

After a transient time of $1.94 \mathrm{~s}$, the estimated message $\hat{u}$ converges to the secret message embedded in the evolution of the transmitter, $u$, as shown in Fig. 5. A low pass Butterworth second order filter was used in order to obtain an accurate chattering-free waveform. The transfer function $H(s)=\frac{1}{s^{2}+1.4142 s+1}$ uses a cutoff frequency $\omega_{c}=30 \mathrm{rad} / \mathrm{s}$. Due to filtering, a delay exists between the two signals. In [Boiko(2005)] an analysis of the high-frequency self-sustained oscillations called chattering in continuous sliding-mode controllers is done.

The estimated state $\hat{x}_{1}$ converges to the state $x_{1}$ of the transmitter after a short time, approximatively $0.06 \mathrm{~s}$. The evolution of the two signals is represented in Fig. 6 and the error $e_{1}=\hat{x}_{1}-x_{1}$ in Fig. 7. Similarly, the estimated state $\hat{x}_{2}$ converges to the state $x_{2}$ of the transmitter after a short time, approximatively $1.65 \mathrm{~s}$, the evolution of the two signals being represented in Fig. 8 and the error $e_{2}=\hat{x}_{2}-x_{2}$ in Fig 9.

- channel affected by additive Gaussian bandlimited white noise.

The considered Gaussian additive band-limited noise has a power of $P_{\text {noise }}=10^{-3} \mathrm{~W}$, when compared to a signal power of $P_{\text {signal }}=1 \mathrm{~W}$, which gives a signal-to-noise ratio of $S N R=$ $30 \mathrm{~dB}$. The measured noisy transmitted output is filtered, using the same filter as the one used for the estimation $\hat{u}$, prior to its input to the sliding-mode observer. Fig. 10 illustrates the $y$ signal at the output of the noisy channel. The estimated message $\hat{u}$ converges to $u$ in 2

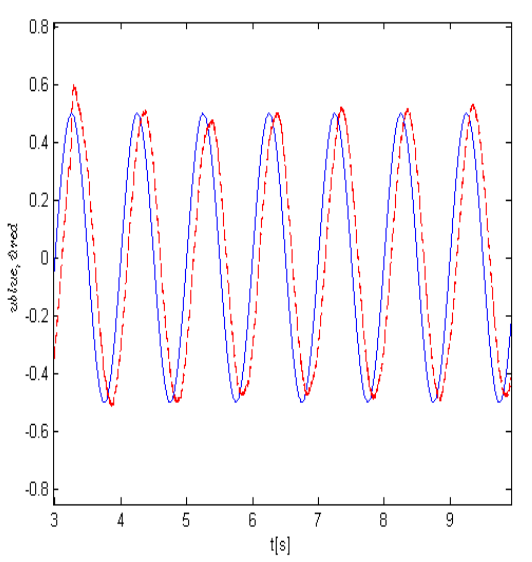

Fig. 5. The estimated $\hat{u}$, in blue, and the transmitted message $u$, in red. The initial conditions are: $x_{1}(0)=0, x_{2}(0)=0.1$ and $x_{2}(t)=0, \forall t \in[-\tau, 0)$.

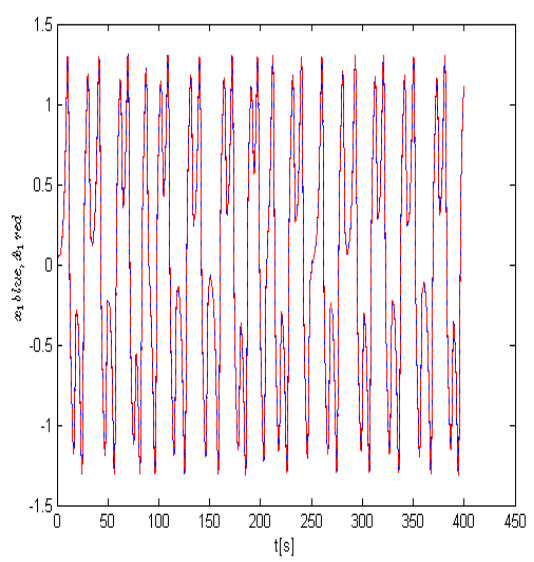

Fig. 6. The estimated $\hat{x}_{1}$ in red, and the state $x_{1}$ in blue. The initial conditions are: $x_{1}(0)=0, x_{2}(0)=0.1$ and $x_{2}(t)=0, \forall t \in$ $[-\tau, 0)$.

$\mathrm{s}$, as shown in Fig. 11. The convergence of $\hat{x}_{1}$ to the state $x_{1}$ is shown in Fig. 12, and the convergence of $\hat{x}_{2}$ to $x_{2}$, in Fig. 13 .

The experimental gain of the observer is $M=$ $10^{4}, e_{1}=\hat{x}_{1}-x_{1}, \nu_{0}$ and $\nu_{1}$ are amplified $K=10^{5}$ times

The presented results stand as proof for the performance of the proposed observer in estimating the states of the transmitter and its unknown input, in the case of a chaotic contin- 


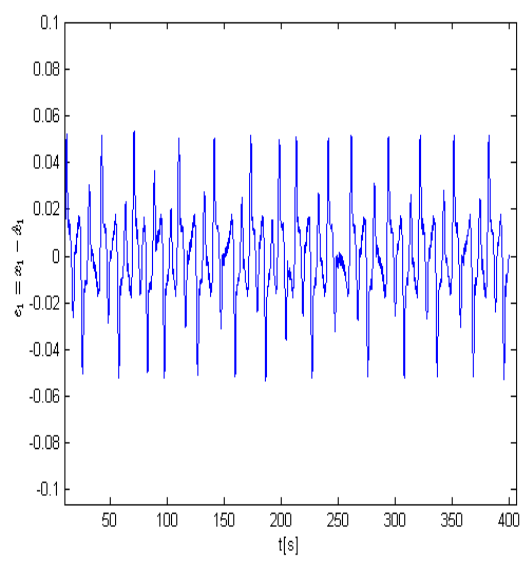

Fig. 7. The error in estimating $x_{1}$.

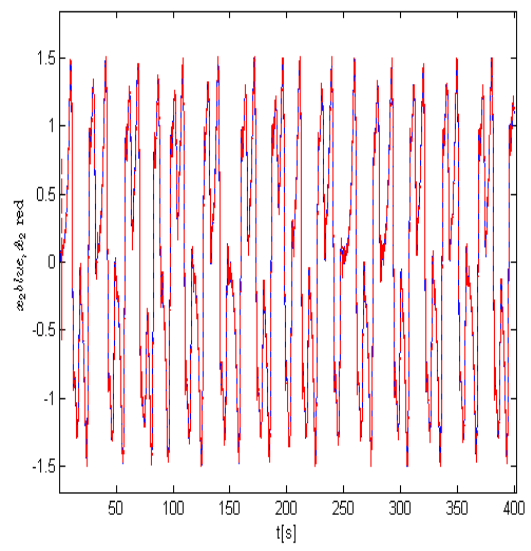

Fig. 8. The estimated $\hat{x}_{2}$ in red, and the state $x_{2}$ in blue. The initial conditions are: $x_{1}(0)=0, x_{2}(0)=0.1$ and $x_{2}(t)=0, \forall t \in$ $[-\tau, 0)$.

uous delayed system, considering the transmission through noise-free channel and through a channel affected by Gaussian additive noise, solving the left invertibility problem these dynamics impose.

\section{CONCLUSION}

In this paper a 3rd-order sliding-mode observer was presented. Its efficiency on the estimation of the states and of the unknown input of a chaotic continuous delayed system was demonstrated. The main contribution of the paper was to propose an observer to solve the left

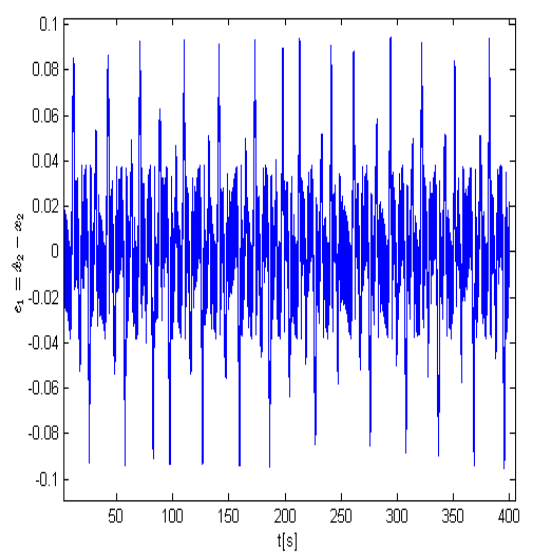

Fig. 9. The error in estimating $x_{2}$.

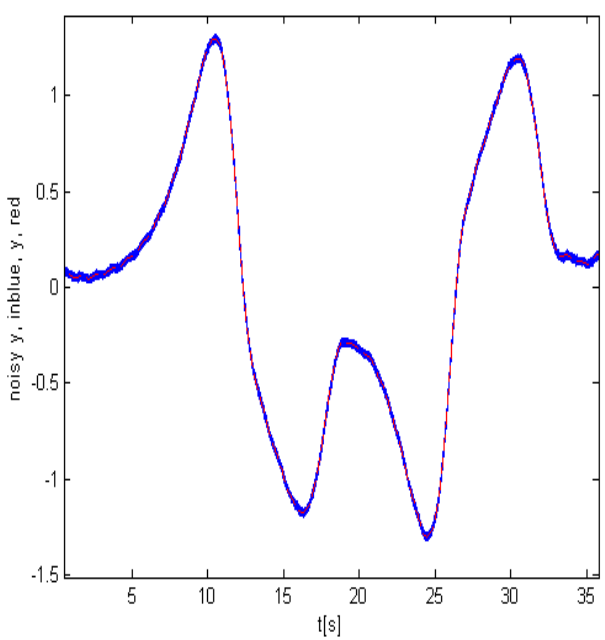

Fig. 10. The noisy y in blue, and y in red.

invertible problem for a particular chaotic delayed system, with and without noisy output measurement. There are many extensions to this preliminary work: the faster and more complicated chaotic delayed transmitter in order to be closer to the secure transmission of real data, analysis of the robustness of such systems to a known plain text attack, conceiving an observer for a chaotic delayed hybrid dynamical system, using the same left invertible technique of high order sliding mode for identification or diagnosis purposes. In a more general context, it may be interesting to synthesize a generic high order sliding mode 


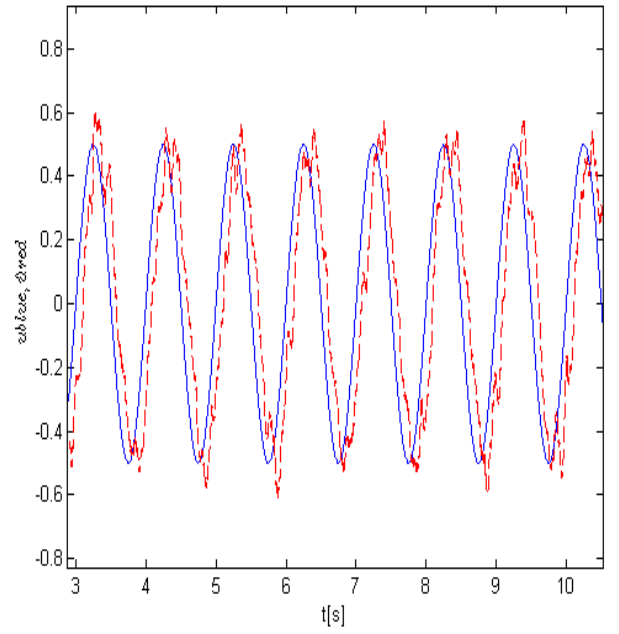

Fig. 11. The estimated $\hat{u}$ in red and the transmitted message $u$ in blue; noisy channel.

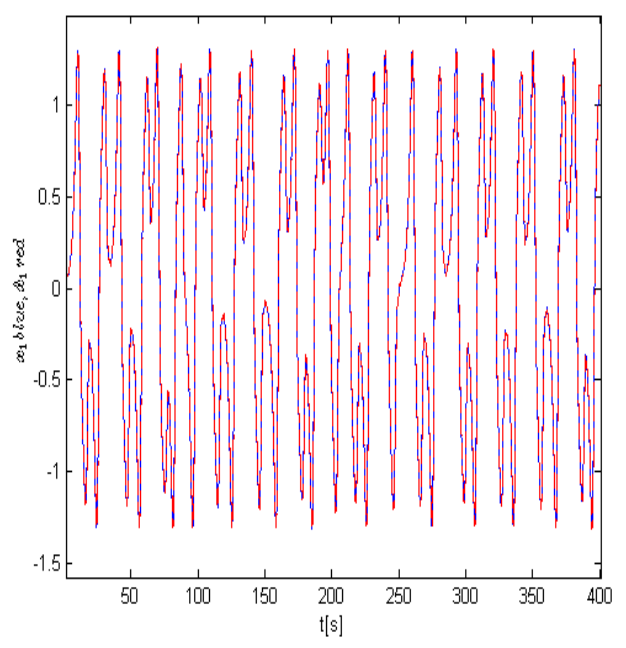

Fig. 12. The estimated $\hat{x}_{1}$ in red, and the state $x_{1}$ in blue; noisy channel.

observer for delayed systems, and to determine under which conditions such an observer works.

\section{REFERENCES}

Angulo, M. T., Fridman, L, and Levant, A. (2011). Robust Exact Finite-Time Output Based Control using High-Order Sliding Modes, Interantional Journal of System Science, volume 42 (11), pp. 1847-1857.

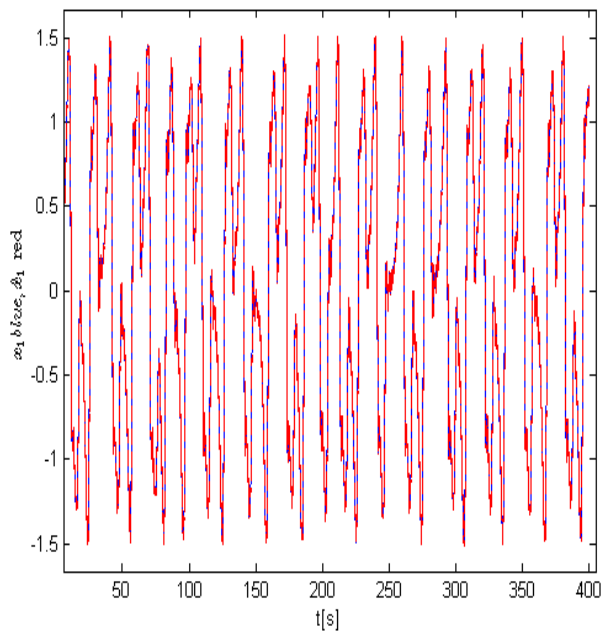

Fig. 13. The estimated $\hat{x}_{2}$ in red, and the state $x_{2}$ in blue; noisy channel.

Boiko, I., Fridman, L. (2005). Analysis of chattering in continuous slidingmode controllers. IEEE Transactions on Automatic Control, volume 50, no.9, pp. 1442-1446.

Djemai, M., and Barbot, J.-P. (2005). New type of data transmission using a synchronization of chaotic systems. International Journal of Bifurcation and Chaos, volume 15, no. 1, pp. 207-223, (c)World Scientific Publishing Company

Levant, A. (2003). Higher-order sliding modes, differentiation and output-feedback control, International Journal on Automatic Control, volume 76, nos 9/10, pp. 924-941.

Levant, A. (2005). Homogeneity approach to high-order sliding mode design.Automatica, volume 41, no. 5, pp. 823-830.

Levant, A.(2006). Exact differentiation of signals with unbounded higher derivatives. Proc. of the 45th IEEE Conference on Decision and Control. pp. 5585-5590.

Uçar, A. (2003). On the chaotic behavior of a prototype delayed dynamical system. Chaos, Solitons and Fractals, 16(2003) 187-194. 